Max-Planck-Institut für demografische Forschung

Max Planck Institute for Demographic Research

Doberaner Strasse 114 - D-18057 Rostock - GERMANY

Tel +49 (0) 3812081 - 0; Fax +49 (0) 3812081 - 202;

http://www.demogr.mpg.de

MPIDR WORKING PAPER WP 2002-042

SEPTEMBER 2002

\title{
Decomposing change in life expectancy: a bouquet of formulas in honour of Nathan Keyfitz's 90th birthday
}

James W. Vaupel (jwv@ demogr.mpg.de)

Vladimir Canudas Romo (canudas@ demogr.mpg.de)

(C) Copyright is held by the authors.

Working papers of the Max Planck Institute for Demographic Research receive only limited review.

Views or opinions expressed in working papers are attributable to the authors and do not necessarily reflect those of the Institute. 


\title{
Decomposing Change in Life Expectancy: A Bouquet of Formulas in Honor of Nathan Keyfitz's $90^{\text {th }}$ Birthday.
}

\author{
J.W. Vaupel V. Canudas Romo \\ Submitted to Demography, August 27, 2002
}

\begin{abstract}
This article extends Nathan Keyfitz's research on continuous change in life expectancy over time. A new formula for decomposing such change is presented and proved. The formula separates change in life expectancy over time into two terms. The first term captures the general effect of reduction in death rates at all ages. The second term captures the effect of heterogeneity in the pace of improvement in mortality at different ages. The formula is extended to decompose change in life expectancy into age-specific and cause-specific components. The methods are applied to analyze changes in life expectancy in Sweden and Japan.
\end{abstract}

\section{Introduction}

Methods to analyze change in life expectancy over time have been developed by various demographers. Pollard (1982, 1988), Arriaga (1984), Pressat (1985) and Andreev (1982) (see Andreev et al. (2002)) focused on the discrete difference in life expectancy at two moments in time. Keyfitz (1977, 1985) considered continuous change and derived a formula that relates the time-derivative of life expectancy to the entropy of lifetable survivorship. 
Mitra (1978), Demetrius (1979), Goldman and Lord (1986), Vaupel (1986), Hokkert (1987) and Hill (1993) further developed this approach.

In this article we present and prove a new decomposition of change in life expectancy over time that generalizes Keyfitz's results. In addition we extend this new method to analyze age-specific and cause-specific effects. We begin with some notation and the proof of the decomposition formula. Then we provide a number of illustrative examples using data for Sweden and Japan.

\section{Preliminaries}

The new decomposition relates the time-derivative of life expectancy to the average pace of improvement in mortality, the average number of life-years lost as a result of death, and the covariance between age-specific rates of mortality improvement and age-specific remaining life expectancies. Before presenting the decomposition it will be useful to briefly discuss each of these quantities.

Life expectancy at birth at time $t$ can be expressed as

$$
e^{o}(0, t)=\int_{0}^{\omega} \ell(a, t) d a,
$$

where $\ell(a, t)$ is the lifetable probability at time $t$ of surviving from birth to age $a$, and $\omega$ is the highest age attained.

It is convenient to use a dot over a variable to denote the derivative with respect to time,

$$
\dot{v} \equiv \dot{v}(a, t) \equiv \frac{\partial}{\partial t} v(a, t),
$$

where $v(a, t)$ is some demographic function. Hence, the time-derivative of life expectancy at birth is expressed as $\dot{e}^{o}(0, t)$.

The force of mortality at age $a$ and at time $t$ is denoted by $\mu(a, t)$. Using an acute accent over the variable to represent the relative derivative or intensity with respect to $t$

$$
\dot{v} \equiv \dot{v}(a, t) \equiv \frac{\dot{v}(a, t)}{v(a, t)} \equiv \frac{\frac{\partial}{\partial t} v(a, t)}{v(a, t)},
$$

we define $\rho(a, t)$ as the rate of progress in reducing death rates,

$$
\rho(a, t)=-\mu(a, t) .
$$


The acute accent notation, which reduces the clutter in many demographic formulas, was originated by Vaupel (1992), and is used in Vaupel and Canudas Romo (2000, 2002).

Let $\bar{v}(t)$ denote the average of $v(a, t)$ over $a$

$$
\bar{v}(t)=\frac{\int_{0}^{\infty} v(a, t) w(a, t) d a}{\int_{0}^{\infty} w(a, t) d a},
$$

where $w(a, t)$ is some weighting function. We define the average improvement in mortality as

$$
\bar{\rho}(t)=\int_{0}^{\omega} \rho(a, t) f(a, t) d a
$$

where $f(a, t)$ is the probability density function describing the distribution of deaths (i.e., lifespans) in the lifetable population at age $a$ and time $t$. Note that in this average the denominator is one, because $\int_{0}^{\omega} f(a, t) d a=1$.

Let $e^{o}(a, t)$ denote remaining life expectancy at age $a$ and time $t$ :

$$
e^{o}(a, t)=\frac{\int_{a}^{\omega} \ell(x, t) d x}{\ell(a, t)} .
$$

Then the average number of life-years lost as a result of death is given by

$$
e^{\dagger}(t)=\int_{0}^{\omega} e^{o}(a, t) f(a, t) d a .
$$

The covariance between functions $u(a, t)$ and $v(a, t)$, with weighting function $w(a, t)$, is

$$
\begin{gathered}
\operatorname{Cov}_{w}(u, v)=\frac{\int_{0}^{\infty}[u(a, t)-\bar{u}(t)][v(a, t)-\bar{v}(t)] w(a, t) d a}{\int_{0}^{\infty} w(a, t) d a} \\
=\frac{\int_{0}^{\infty} u(a, t) v(a, t) w(a, t) d a}{\int_{0}^{\infty} w(a, t) d a}-\frac{\int_{0}^{\infty} u(a, t) w(a, t) d a}{\int_{0}^{\infty} w(a, t) d a} \frac{\int_{0}^{\infty} v(a, t) w(a, t) d a}{\int_{0}^{\infty} w(a, t) d a} \\
\equiv \overline{u v}-\bar{u} \bar{v} .
\end{gathered}
$$

Hence, the covariance between age-specific rates of mortality improvement and age-specific remaining life expectancies, weighted by the distribution of deaths, is

$$
\operatorname{Cov}_{f}\left(\rho, e^{o}\right)=\int_{0}^{\omega}[\rho(a, t)-\bar{\rho}(t)]\left[e^{o}(a, t)-e^{\dagger}(t)\right] f(a, t) d a .
$$


Note that formula (9) implies that the expectation of a product can be decomposed as

$$
\overline{u v}=\bar{u} \bar{v}+\operatorname{Cov}_{w}(u, v),
$$

a result central to our derivation.

\section{A New Decomposition of the Time- Derivative of Life Expectancy}

Change in life expectancy can be decomposed as follows:

$$
\dot{e}^{o}(0, t)=\bar{\rho} e^{\dagger}+\operatorname{Cov}_{f}\left(\rho, e^{o}\right) .
$$

Proof

From the definition of life expectancy in formula (1) and the fact that $\ell(a, t)=e^{-\int_{0}^{a} \mu(x, t) d x}$, it follows that the time-derivative of life expectancy is

$$
\dot{e}^{o}(0, t)=\int_{0}^{\omega} \dot{\ell}(a, t) d a=\int_{0}^{\omega} \ell(a, t) \dot{\ell}(a, t) d a=-\int_{0}^{\omega} \ell(a, t) \int_{0}^{a} \dot{\mu}(x, t) d x d a .
$$

In terms of the rate of progress in reducing death rates, $\rho(a, t)=-\mu(a, t)$, the derivative of life expectancy can be expressed as

$$
\dot{e}^{o}(0, t)=\int_{0}^{\omega} \ell(a, t) \int_{0}^{a} \mu(x, t) \rho(x, t) d x d a=\int_{0}^{\omega} \mu(a, t) \rho(a, t) \int_{a}^{\omega} \ell(x, t) d x d a .
$$

See Goldman and Lord (1986) and Vaupel (1986) for further discussion of the reversal of integration used to derive (14). Given formula (7) for the remaining expectation of life at age $a$ and time $t, e^{o}(a, t)$, and the probability density function describing the distribution of deaths $f(a, t)=\mu(a, t) \ell(a, t)$, formula (14) implies that

$$
\dot{e}^{o}(0, t)=\int_{0}^{\omega} \mu(a, t) \rho(a, t) \ell(a, t) e^{o}(a, t) d a=\int_{0}^{\omega} \rho(a, t) e^{o}(a, t) f(a, t) d a .
$$

Formula (15) can be decomposed using (11), the formula for the expectation of a product:

$$
\dot{e}^{o}(0, t)=\bar{\rho} e^{\dagger}+\operatorname{Cov}_{f}\left(\rho, e^{o}\right) .
$$

Q.E.D. 
The decomposition in formula (12) expresses the change in life expectancy at birth as the sum of two terms. The first term is the product of the average rate of mortality improvement and the average number of life-years lost. This term captures the general effect of a reduction in death rates and will be called the "level-1 change" in this article. Note that $\bar{\rho}$ can be interpreted as the proportion of deaths averted (or lives saved) and $e^{\dagger}$ can be interpreted as the average number of life-years gained per life saved.

The second term, the covariance between rates of mortality improvement and remaining life expectancies, increases or decreases the general effect, depending on whether the covariance is positive or negative. If $\rho(a, t)$ is constant at all ages, then the covariance is zero. Hence, the covariance captures the effect of heterogeneity in $\rho(a, t)$ at different ages. If the pace of mortality improvement tends to be greatest at ages at which remaining life expectancy is long, then the covariance will be positive. The covariance term will be called the "level-2 change" in this article. Another decomposition without the covariance term can be seen in Note 4 in this article.

Formula (12) is analogous to the decomposition of Vaupel and Canudas Romo (2002). That formula breaks the change in an average into a level1 term involving the average of age-specific changes and a level-2, covariance term that captures the effect due to heterogeneity in age-specific or subpopulation-specific changes.

\section{An Illustration: Change in Swedish Life Expectancy}

Table 1 shows the application of formula (12) to the annual change in life expectancy at birth for the Swedish population in 1903, 1953 and 1998.

Over the course of the $20^{\text {th }}$ century Swedish life expectancy increased substantially. The average pace of mortality improvement, $\bar{\rho}$, fluctuated from about $1.9 \%$ at the turn of the century to $2.1 \%$ at mid century and $1.6 \%$ at the end of the century. The average number of life-years lost as a result of death, $e^{\dagger}$, dropped from 22 years in 1903 to around 12 years in 1950 and 10 years in 1998. The product $\bar{\rho} e^{\dagger}$ describes the increase in life expectancy due to the general advance in survivorship. This level-1 component is positive and is the main contributor to the increase in life expectancy.

The level-2 component is the covariance between age-specific improve- 
Table 1. Life expectancy at birth, $e^{o}(0, t)$, and the decomposition of the annual change around the first of January of 1903, 1953 and 1998, in Sweden.

\begin{tabular}{lrrr}
\hline$t$ & 1903 & \multicolumn{1}{c}{1953} & \multicolumn{1}{c}{1998} \\
\hline$e^{o}(0, t-2.5)$ & 52.239 & 71.130 & 78.784 \\
$e^{o}(0, t+2.5)$ & 54.527 & 72.586 & 79.740 \\
$\dot{e}^{o}(0, t)$ & 0.458 & 0.291 & 0.191 \\
& & & \\
$\bar{\rho}(\%)$ & 1.852 & 2.083 & 1.587 \\
$e^{\dagger}$ & 22.362 & 11.988 & 10.053 \\
$\bar{\rho} e^{\dagger}$ & 0.414 & 0.249 & 0.159 \\
$\operatorname{Cov}_{f}\left(\rho, e^{o}\right)$ & 0.044 & 0.042 & 0.032 \\
$\dot{e}^{o}(0)=\bar{\rho} e^{\dagger}+\operatorname{Cov}_{f}\left(\rho, e^{o}\right)$ & 0.458 & 0.291 & 0.191 \\
\hline
\end{tabular}

Source: Authors' calculations described in Note 1 and Note 2. Life table data is derived from the Human Mortality Database (2002). Life table values for the years 1900 and 1905, 1950 and 1955, 1995 and 2000, were used to obtain results for the mid-points around January 1, 1903, 1953 and 1998.

ments in mortality and remaining life expectancies. This term is positive but relatively small. It is positive because relatively large reductions in mortality were achieved at ages with relatively long remaining life expectancies.

\section{Relationship to the Entropy of the Survival Function}

Following Keyfitz (1985), let $\mathcal{H}(t)$ denote the entropy of the survival function

$$
\mathcal{H}(t)=-\frac{\int_{0}^{\omega} \ell(a, t) \ln [\ell(a, t)] d a}{\int_{0}^{\omega} \ell(a, t) d a} .
$$

Goldman and Lord (1986), and Vaupel (1986) show that this entropy can also be expressed as

$$
\mathcal{H}(t)=\frac{\int_{0}^{\omega} \ell(a, t) \int_{0}^{a} \mu(x, t) d x d a}{\int_{0}^{\omega} \ell(a, t) d a}
$$




$$
\begin{gathered}
=\frac{\int_{0}^{\omega} \int_{0}^{a} \ell(a, t) \mu(x, t) d x d a}{\int_{0}^{\omega} \ell(a, t) d a}=\frac{\int_{0}^{\omega} \int_{x}^{\omega} \ell(a, t) \mu(x, t) d a d x}{e^{o}(0, t)} \\
=\frac{\int_{0}^{\omega} \mu(x, t) \int_{x}^{\omega} \ell(a, t) d a d x}{e^{o}(0, t)}=\frac{\int_{0}^{\omega} \mu(x, t) \ell(x, t) e^{o}(x, t) d x}{e^{o}(0, t)} .
\end{gathered}
$$

Given formula (8) for $e^{\dagger}$ it follows that

$$
e^{\dagger}(t)=e^{o}(0, t) \mathcal{H}(t)
$$

If $\rho(a, t)$ is constant over age, $\rho(a, t)=\rho(t)$ for all $a$, then (12) reduces to

$$
\dot{e}^{o}(0, t)=\rho(t) e^{\dagger}(t) .
$$

Substituting (18) yields

$$
\dot{e}^{o}(0, t)=\rho(t) \mathcal{H}(t) e^{o}(0, t),
$$

or

$$
e^{o}(0, t)=\frac{\dot{e}^{o}(0, t)}{e^{o}(0, t)}=\rho(t) \mathcal{H}(t),
$$

which was Keyfitz's (1985) main result. Note that in (20) the change depends not only on mortality progress and the entropy $\mathcal{H}(t)$, but also on the level of life expectancy $e^{o}(0, t)$.

If $\rho(a, t)$ varies with age, then (18) implies that our main result (12) can be re-expressed as

$$
\dot{e}^{o}(0, t)=\bar{\rho}(t) \mathcal{H}(t) e^{o}(0, t)+\operatorname{Cov}_{f}\left(\rho, e^{o}\right),
$$

or, alternatively, the relative change in life expectancy can be decomposed as

$$
\dot{e}^{o}(0, t)=\frac{\dot{e}^{o}(0, t)}{e^{o}(0, t)}=\bar{\rho}(t) \mathcal{H}(t)+\frac{\operatorname{Cov}_{f}\left(\rho, e^{o}\right)}{e^{o}(0, t)} .
$$

This result generalizes Keyfitz's result in (21).

If mortality follows a shifting Gompertz trajectory with changing level but constant rate of increase,

$$
\mu(a, t)=\mu(0, t) e^{b a},
$$

then Vaupel ((1986), see also Vaupel and Canudas Romo (2000)) proved that

$$
e^{o}(0, t) \mathcal{H}(t) \approx \frac{1}{b} .
$$


From formula (18) it follows that the average life expectancy lost due to death is

$$
e^{\dagger}(t) \approx \frac{1}{b}
$$

The value of $b$ can be estimated from the slope of a regression line fitted to the logarithm of age-specific death rates from age 30 to 95 years, the span of life when mortality approximately follows a Gompertz trajectory. For Sweden in 1900, 1950 and 2000, the values of $\frac{1}{b}$ were around $13.803,10.480$ and 10.127, respectively. For the same years, the average number of life-years lost as a result of death after age $30, e_{30}^{\dagger}(t)$, are 12.382, 10.012 and 10.010. (Note that even though all the formulas presented here are for life expectancy at birth, they can also be used at any other age, by using a lifetable starting at that age.)

\section{Age Decomposition}

Formula (15) can be decomposed by age category as follows

$$
\begin{gathered}
\dot{e}^{o}(0, t)=\int_{0}^{\omega} \rho(a, t) e^{o}(a, t) f(a, t) d a \\
=\int_{0}^{x_{1}} \rho(a, t) e^{o}(a, t) f(a, t) d a+\ldots+\int_{x_{n}}^{\omega} \rho(a, t) e^{o}(a, t) f(a, t) d a \\
=\frac{\int_{0}^{x_{1}} \rho(a, t) e^{o}(a, t) f(a, t) d a}{\int_{0}^{x_{1}} f(a, t) d a} \int_{0}^{x_{1}} f(a, t) d a+ \\
\ldots+\frac{\int_{x_{n}}^{\omega} \rho(a, t) e^{o}(a, t) f(a, t) d a}{\int_{x_{n}}^{\omega} f(a, t) d a} \int_{x_{n}}^{\omega} f(a, t) d a .
\end{gathered}
$$

The averages in this formula can be decomposed using (11), the formula for the expectation of a product. For the age group $x_{i}$ to $x_{i+1}$

$$
\frac{\int_{x_{i}}^{x_{i+1}} \rho(a, t) e^{o}(a, t) f(a, t) d a}{\int_{x_{i}}^{x_{i+1}} f(a, t) d a}=[\bar{\rho}]_{x_{i}}^{x_{i+1}}\left[e^{\dagger}\right]_{x_{i}}^{x_{i+1}}+\left[\operatorname{Cov}_{f}\left(\rho, e^{o}\right)\right]_{x_{i}}^{x_{i+1}},
$$

where $[\bar{\rho}]_{x_{i}}^{x_{i+1}}$ is average improvement in mortality in the age group $x_{i}$ to $x_{i+1}$,

$$
[\bar{\rho}]_{x_{i}}^{x_{i+1}}=\frac{\int_{x_{i}}^{x_{i+1}} \rho(a, t) f(a, t) d a}{\int_{x_{i}}^{x_{i+1}} f(a, t) d a}
$$


the number of life-years lost as a result of death $\left[e^{\dagger}\right]_{x_{i}}^{x_{i+1}}$ in the age group $x_{i}$ to $x_{i+1}$ is defined as

$$
\left[e^{\dagger}\right]_{x_{i}}^{x_{i+1}}=\frac{\int_{x_{i}}^{x_{i+1}} e^{o}(a, t) f(a, t) d a}{\int_{x_{i}}^{x_{i+1}} f(a, t) d a}
$$

and the component of the covariance in the age group $x_{i}$ to $x_{i+1}$ is defined as

$$
\left[\operatorname{Cov}\left(\rho, e^{o}\right)\right]_{x_{i}}^{x_{i+1}}=\frac{\int_{x_{i}}^{x_{i+1}}[\rho(a, t)-\bar{\rho}(t)]\left[e^{o}(a, t)-e^{\dagger}(t)\right] f(a, t) d a}{\int_{x_{i}}^{x_{i+1}} f(a, t) d a},
$$

where $\bar{\rho}(t)$ and $e^{\dagger}(t)$ are as defined in formulas (29) and (30).

If the age category is narrow enough, then $\rho(a, t)$ and $e^{o}(a, t)$ will not vary much within the age category. This implies that the covariance terms will be close to zero. Hence we have the approximation

$$
\begin{aligned}
\dot{e}^{o}(0, t) & \approx\left[\dot{e}^{o}\right]_{0}^{x_{1}}+\ldots+\left[\dot{e}^{o}\right]_{x_{n}}^{\omega} \\
& =[\bar{\rho}]_{0}^{x_{1}}\left[e^{\dagger}\right]_{0}^{x_{1}}[F]_{0}^{x_{1}}+\ldots+[\bar{\rho}]_{x_{n}}^{\omega}\left[e^{\dagger}\right]_{x_{n}}^{\omega}[F]_{x_{n}}^{\omega},
\end{aligned}
$$

where $[F]_{x_{i}}^{x_{i+1}}$ denotes the distribution of deaths in the age group $x_{i}$ to $x_{i+1}$

$$
[F]_{x_{i}}^{x_{i+1}}=\int_{x_{i}}^{x_{i+1}} f(a, t) d a .
$$

For single years of age this approximation will be very good. It can be written as

$$
\begin{aligned}
\dot{e}^{o}(0, t) \approx & {\left[\dot{e}^{o}\right]_{0}+\left[\dot{e}^{o}\right]_{1}+\ldots+\left[\dot{e}^{o}\right]_{\omega} } \\
= & \rho(0.5, t) e^{o}(0.5, t) f(0.5, t)+\rho(1.5, t) e^{o}(1.5, t) f(1.5, t)+ \\
& \ldots+\rho(\omega-0.5, t) e^{o}(\omega-0.5, t) f(\omega-0.5, t)
\end{aligned}
$$

with the understanding that $\rho(i+1 / 2, t)$ is the rate of progress in reducing mortality, $e^{o}(i+1 / 2, t)$ is the remaining life expectancy, and $f(i+1 / 2, t)$ is the proportion of deaths, between exact age $i$ and $i+1$.

The three age-specific components of formula (32) are shown in Figures 1, 2 and 3 for Sweden in 1903, 1953 and 1998. Figure 4 shows the value of the resulting age-specific components of the change in life expectancy, the $\left[\dot{e}^{o}\right]_{a}$ terms. Because the value of this component was so large at ages 0,1 and 2 in 1903, the Figure is restricted to ages 5 and older. In 1903, fully 55\% of the change in life expectancy was due to mortality change at age $0-2$. 
Figure 1. Five-year moving average of the improvement in mortality at ages 2 to 99 for Sweden in 1903, 1953 and 1998.

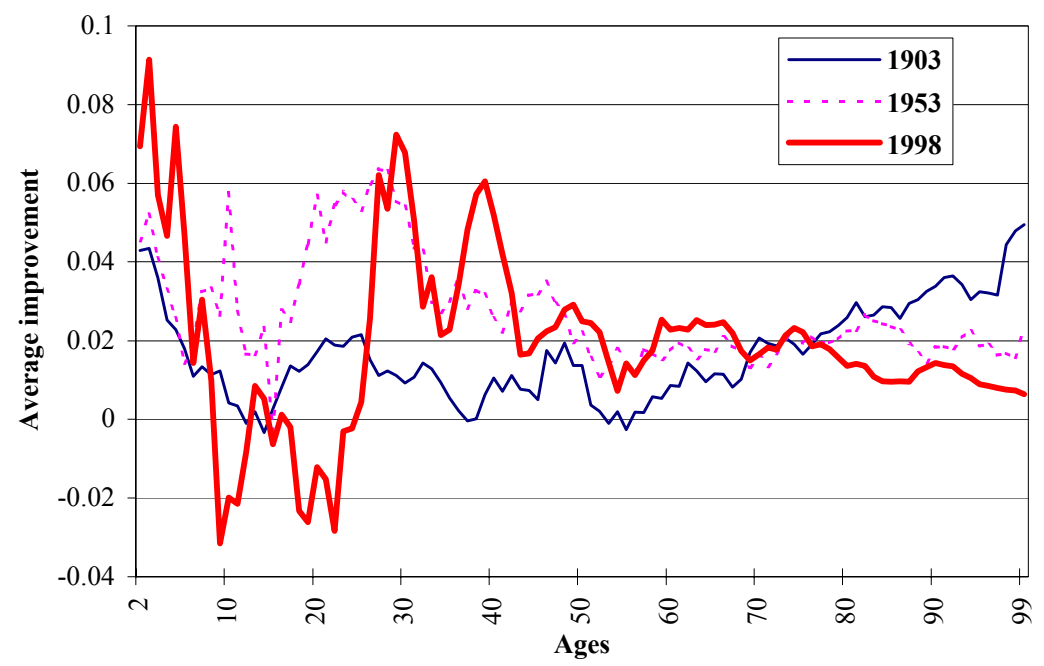

Figure 2. Remaining life expectancy at ages 0 to 99 for Sweden in 1903, 1953 and 1998.

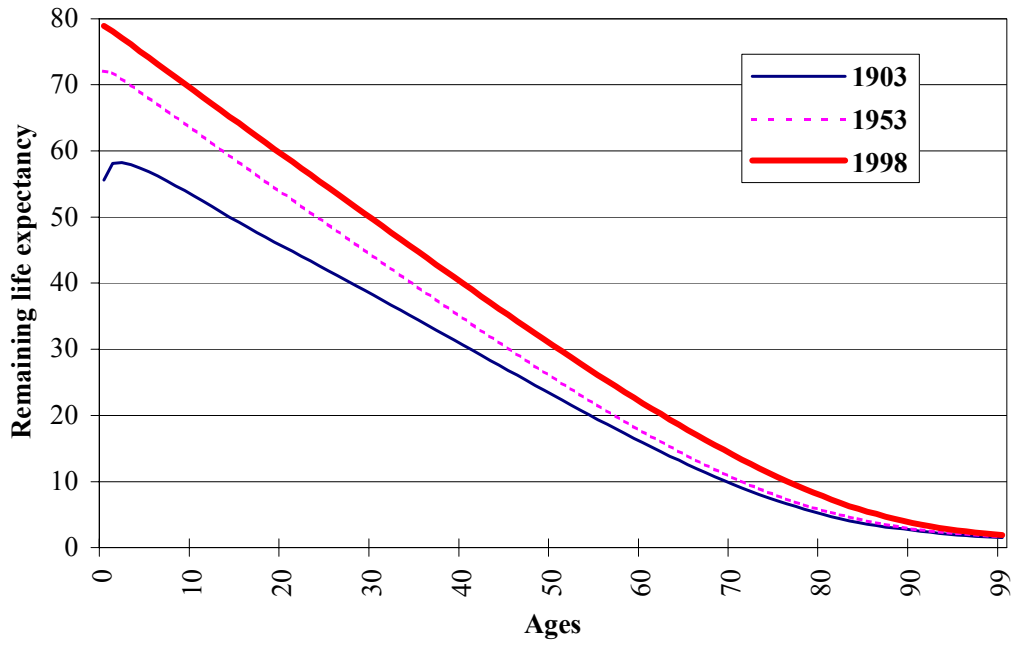


Figure 3. Distribution of deaths at ages 0 to 99 for Sweden in 1903, 1953 and 1998.

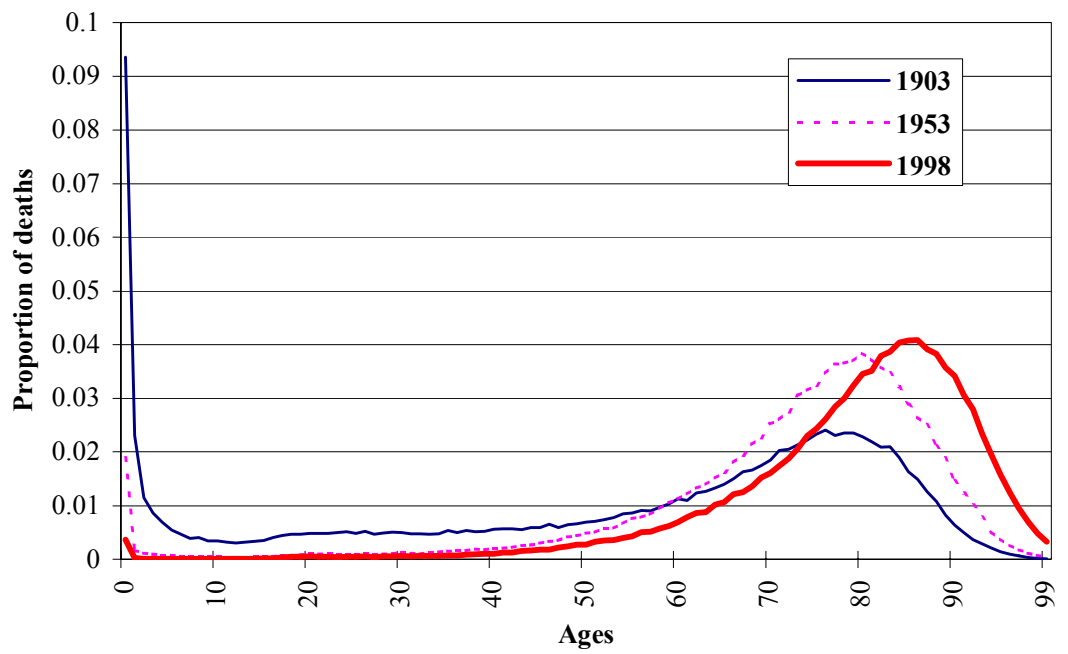

Figure 4. Five-year moving average of the age contribution to the change in life expectancy at ages 5 to 99 for Sweden in 1903, 1953 and 1998.

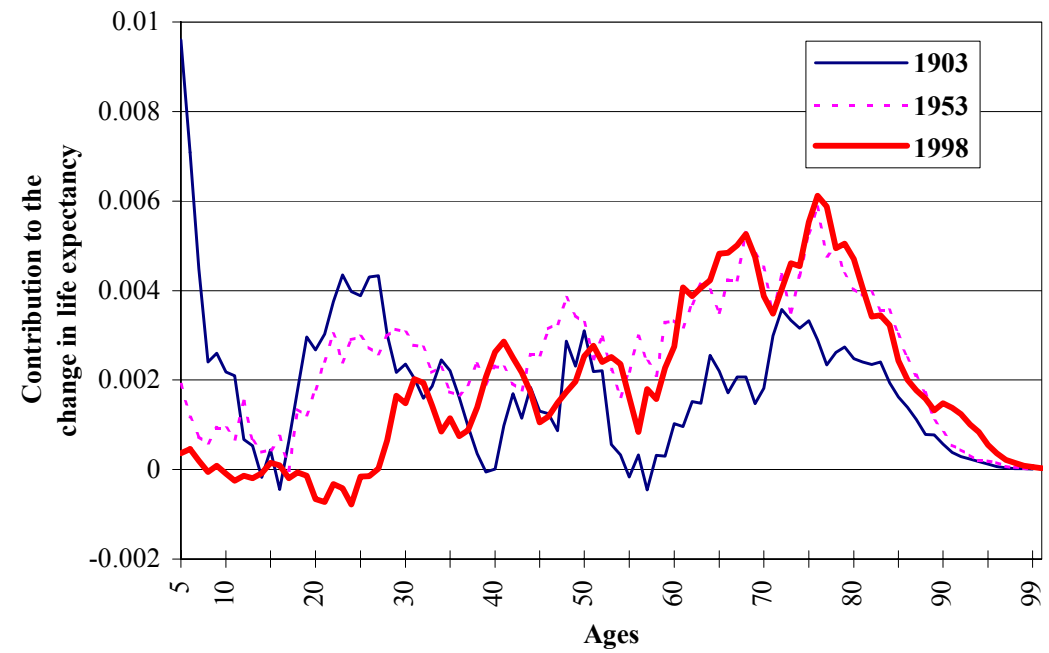




\section{Decomposing Life Expectancy by Cause of Death}

Let $\mu_{i}(a, t)$ be the force of mortality from cause of death $i$ at age $a$ and time $t$. The chance of surviving, i.e., not dying from cause $i$, is then $\ell_{i}(a, t)=$ $e^{-\int_{0}^{a} \mu_{i}(x, t) d x}$. For competing, independent causes of death $\ell(a, t)=\ell_{1}(a, t) \ldots \ell_{n}(a, t)$.

Hence,

$$
e^{o}(0, t)=\int_{0}^{\omega} \ell(a, t) d a=\int_{0}^{\omega} \ell_{1}(a, t) \ldots \ell_{n}(a, t) d a,
$$

and

$$
\begin{aligned}
\dot{e}^{o}(0, t) & =\int_{0}^{\omega} \dot{\ell}_{1}(a, t) \ldots \ell_{n}(a, t) d a+\ldots+\int_{0}^{\omega} \ell_{1}(a, t) \ldots \dot{\ell}_{n}(a, t) d a \\
& =\int_{0}^{\omega} \dot{\ell}_{1}(a, t) \ell_{1}(a, t) \ldots \ell_{n}(a, t) d a+\ldots+\int_{0}^{\omega} \dot{\ell}_{n}(a, t) \ell_{1}(a, t) \ldots \ell_{n}(a, t) d a \\
& =\int_{0}^{\omega} \dot{\ell}_{1}(a, t) \ell(a, t) d a+\ldots+\int_{0}^{\omega} \dot{\ell}_{n}(a, t) \ell(a, t) d a .
\end{aligned}
$$

Each of the terms in (36) can be reexpressed, using the same logic as explained above:

$$
\begin{gathered}
\int_{0}^{\omega} \ell(a, t) \dot{\ell}_{i}(a, t) d a=-\int_{0}^{\omega} \ell(a, t) \int_{0}^{a} \dot{\mu}_{i}(x, t) d x d a \\
=-\int_{0}^{\omega} \dot{\mu}_{i}(a, t) \int_{a}^{\omega} \ell(x, t) d x d a=-\int_{0}^{\omega} \dot{\mu}_{i}(a, t) \ell(a, t) e^{o}(a, t) d a .
\end{gathered}
$$

Thus

$$
\dot{e}^{o}(0, t)=-\sum_{i=1}^{n} \int_{0}^{\omega} \dot{\mu}_{i}(a, t) \ell(a, t) e^{o}(a, t) d a .
$$

This formula is the continuous version of the discrete difference formula presented by Pollard $(1982,1988)$.

Let $\rho_{i}(a, t)$ denote the pace of reduction of mortality from cause $i, \rho_{i}(a, t)=$ $-\mu_{i}(a, t)$. The proportion of deaths from cause $i$ at age $a$ and time $t$ is $f_{i}(a, t)=\mu_{i}(a, t) \ell(a, t)$. It then follows from (37) that

$$
\dot{e}^{o}(0)=\sum_{i=1}^{n} \int_{0}^{\omega} \rho_{i}(a, t) e^{o}(a, t) f_{i}(a, t) d a .
$$

Applying the decomposition in formula (11) yields

$$
\dot{e}^{o}(0, t)=\sum_{i=1}^{n}\left[\bar{\rho}_{i} e_{i}^{\dagger}+\operatorname{Cov}_{f_{i}}\left(\rho_{i}, e^{o}\right)\right] F_{i},
$$


where

$$
F_{i} \equiv F_{i}(t)=\int_{0}^{\omega} f_{i}(a, t) d a,
$$

$\bar{\rho}_{i}(t)$ is the average pace of reduction of mortality from cause $i$ :

$$
\bar{\rho}_{i}(t)=\frac{\int_{0}^{\omega} \rho_{i}(a, t) f_{i}(a, t) d a}{\int_{0}^{\omega} f_{i}(a, t) d a}
$$

$e_{i}^{\dagger}(t)$ is the average number of life-years lost as a result of cause of death $i$,

$$
e_{i}^{\dagger}(t)=\frac{\int_{0}^{\omega} e^{o}(a, t) f_{i}(a, t) d a}{\int_{0}^{\omega} f_{i}(a, t) d a}
$$

and the covariance is between the rate of improvement in mortality from cause of death $i$ and the remaining life expectancy at various ages

$$
\operatorname{Cov}_{f_{i}}\left(\rho_{i}, e^{o}\right)=\frac{\int_{0}^{\omega}\left[\rho_{i}(a, t)-\bar{\rho}_{i}(t)\right]\left[e^{o}(a, t)-e_{i}^{\dagger}(t)\right] f_{i}(a, t) d a}{\int_{0}^{\omega} f_{i}(a, t) d a} .
$$

Note that the averages (6) to (10) differ from those (41) to (43), because in the latter equations the denominators do not add to one, $F_{i}(t)=\int_{0}^{\omega} f_{i}(a, t) d a \neq$ 1 .

\section{An Illustration: Change in Japanese Life Expectancy}

Take the case of Japan as an example: The lifetable distribution of deaths due to the different causes of death in 1980 and 1990 is shown in Table 2. This is a distribution of causes of death for a lifetable population in which the proportion of people at each age is determined by lifetable probabilities of survival.

Table 3 and Figure 5 present the results of applying the decomposition formula in (39) to the Japanese data. Over the decade from 1980 to 1990, Japanese life expectancy rose from 75.91 to 78.80 years, with an estimated annual increase of $\dot{e}^{o}(0,1985)=0.288$. As shown in Table 3 and Figure 5, three fifths of this increase in life expectancy can be attributed to a reduction in mortality due to cerebrovascular disease and heart disease. 
Table 2. Lifetable distribution of causes of death for Japan in 1980 and 1990.

\begin{tabular}{lrr}
\hline Cause of Death & \multicolumn{1}{c}{1980} & \multicolumn{1}{c}{1990} \\
& \multicolumn{1}{c}{$\%$} \\
\hline Heart disease & 21.4 & 23.7 \\
Malignant neoplasm & 18.5 & 21.6 \\
Cerebrovascular disease & 24.3 & 16.1 \\
Infectious diseases & 8.6 & 12.8 \\
Violent deaths & 4.6 & 4.5 \\
Stomach, liver and & & \\
kidney disorders & 4.3 & 4.3 \\
Senility without psychosis & 7.4 & 5.0 \\
Other causes & 10.9 & 12.0 \\
\hline All causes of death & 100.00 & 100.00 \\
\hline
\end{tabular}

Source: Authors' calculations described in Note 1 and 2, based on the Berkeley Mortality Database (2001). Heart disease includes hypertensive disease. Other causes of death are those denoted in the Berkeley Mortality Database (2001) as Other Causes, plus congenital malformations and diabetes mellitus. Infectious diseases include pneumonia and bronchitis.

On average, death rates from malignant neoplasms and infectious diseases increased, yielding negative values of $\bar{\rho}$ and negative level-1 changes. As opposed to this, the level- 2 changes for these causes of death had positive values, because improvements were made at younger ages with high remaining life expectancy. As a result of the balance between level-1 and level-2 change, the final column of Table 3 shows only positive contributions for all the causes of death.

\section{Relationship to the Cause-Specific Entropy of the Survival Function}

Keyfitz (1977) derived a formula to study the effects that health improvements have on the change of life expectancy over time

$$
e^{o}(0, t)=\sum_{i=1}^{n} \rho_{i}(t) \mathcal{H}_{i}(t),
$$


Table 3. Cause of death decomposition for the annual change over time in life expectancy, for Japan around January 1, 1985.

\begin{tabular}{lcccccc}
\hline Cause of Death & $\bar{\rho}_{i}(\%)$ & $e_{i}^{\dagger}$ & $\bar{\rho}_{i} e_{i}^{\dagger}$ & $\operatorname{Cov}_{f_{i}}\left(\rho_{i}, e^{o}\right)$ & $F_{i}(\%)$ & $\dot{e}_{i}^{o}(0)$ \\
\hline Heart disease & 2.058 & 8.333 & 0.172 & 0.022 & 22.543 & 0.044 \\
$\begin{array}{l}\text { Malignant neoplasm } \\
\text { Cerebrovascular }\end{array}$ & -0.098 & 13.276 & -0.013 & 0.088 & 20.042 & 0.015 \\
disease & & & & & & \\
$\begin{array}{l}\text { Infectious diseases } \\
\text { Violent deaths }\end{array}$ & -0.979 & 8.594 & 0.600 & 0.038 & 20.226 & 0.129 \\
$\begin{array}{l}\text { Stomach, liver and } \\
\text { kidney disorders }\end{array}$ & 1.608 & 23.942 & -0.056 & 0.104 & 10.718 & 0.005 \\
$\begin{array}{l}\text { Senility without } \\
\text { psychosis }\end{array}$ & 2.168 & 11.548 & 0.376 & 0.058 & 4.516 & 0.020 \\
Other causes & 9.379 & 4.294 & 0.403 & 0.094 & 4.294 & 0.015 \\
\hline All causes of death & 1.432 & 13.792 & 0.197 & 0.137 & 11.461 & 0.038 \\
\hline
\end{tabular}

Source: Authors' calculations described in Note 1 and 2, based on the Berkeley Mortality Database (2001).

where $\rho_{i}(t)$ represents the pace of the improvement, which is assumed to be the same at all ages, at time $t$ for the $i$ th cause of death, and $\mathcal{H}_{i}(t)$ the entropy of the $i$ th cause of death,

$$
\mathcal{H}_{i}(t)=-\frac{\int_{0}^{\omega} \ell(a, t) \ln \left[\ell_{i}(a, t)\right] d a}{\int_{0}^{\omega} \ell(a, t) d a} .
$$

In Keyfitz's words, this entropy is "a measure of the length of time from the nondeath from the $i$ th cause up to the time when the person dies from the next thing that will hit him" (1977: 414).

The entropy of the $i$ th cause of death and the average number of life-years lost as a result of cause of death $i$ are related by

$$
e_{i}^{\dagger}(t)=\frac{\mathcal{H}_{i}(t) e^{o}(0, t)}{F_{i}(t)}
$$

This result can be derived in the same way as formula (23). Substituting 
Figure 5. Cause of death decomposition for the annual change over time in life expectancy, for Japan around January 1, 1985.

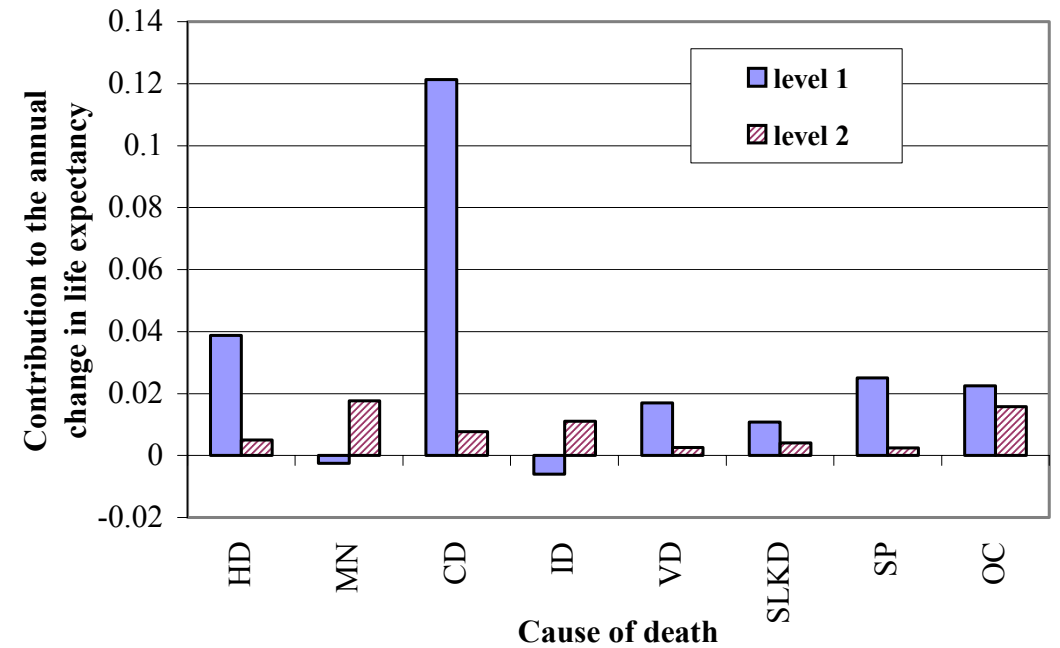

Note: The abbreviations correspond to: HD-Heart disease; MN- Malignant neoplasm; CD-Cerebrovascular disease; ID-Infectious diseases; VD-Violent deaths; SLKDStomach, liver and kidney disorders; SP-Senility without psychosis; OC-Other causes.

formula (46) in (39), and dividing by the life expectancy yields

$$
e^{o}(0, t)=\sum_{i=1}^{n}\left(\bar{\rho}_{i} \mathcal{H}_{i}(t)+\frac{\operatorname{Cov}_{f_{i}}\left(\rho_{i}, e^{o}\right) F_{i}}{e^{o}(0, t)}\right),
$$

which generalizes Keyfitz's formula (44) to the case when rates of reduction in cause-specific mortality can vary from age to age.

\section{Discussion}

Over the past two decades decomposition of change in life expectancy has been a mainstay of demographic analysis. Almost all the many applications have concerned discrete changes in life expectancy, with Arriaga's (1984) formulation being particularly popular. Keyfitz's research on time-derivatives 
of life expectancy has largely been of theoretical interest because of the restrictive, unrealistic assumption that the pace of mortality change is constant at all ages.

Reality is continuous and calculus is elegant, but data are discrete. In this article we derive decomposition formulas for time-derivatives of life expectancy: our formulas involve derivatives and integrals. Pollard $(1982,1988)$ studied discrete differences in life expectancy between two points in time, using formulas that involve integrals over age. Arriaga (1984) analyzed discrete differences in life expectancy using formulas that take sums over age. All three approaches are closely related when applied to actual data pertaining to time intervals of a few years. Depending on the approach, either the formulas or the estimation procedures involve approximations or inelegancies. Pollard (1988) explained the underlying similarly of his method to Arriaga's and his method can also be shown to be similar, in empirical applications, to ours. Hence it is no surprise that Arriaga's method for decomposing change in life expectancy by age yields the same results as the Vaupel-Canudas method for Sweden around 1998, as shown in Table 4. Similarly, decomposition of change in life expectancy by cause of death using traditional methods will generally produce essentially the same results as our new method. Why, then, should demographers consider the methods developed in this article? There are three main reasons.

First, our method permits further decomposition of age-specific and causespecific effects into the effects - for each age category or for each cause of death - of the pace of mortality improvement, remaining life expectancy, and the frequency of deaths.

Second, our method permits decomposition of change in life expectancy into the general impact of mortality improvement at all ages (our "level-1 effect") and the additional effect of heterogeneity in the age-specific rates of improvement (our "level-2" effect). The general impact can be further decomposed into the average rate of mortality improvement multiplied by the average number of life-years saved. We conjecture that this kind of decomposition will lead to more interesting demographic insights than Arriaga's distinction between the direct and indirect effects of mortality improvements.

Third, our formulas are both elegant and exact. It is necessary to use approximations when applying them to data, which is a minor drawback. Because the formulas are elegant, they aid understanding and permit deeper comprehension of the demographic factors that are driving change in life expectancy. The formulas are thus in the spirit of Nathan Keyfitz's enduring 
Table 4. Age decomposition of the annual change over time in life expectancy using Arriaga's and Vaupel-Canudas' decompositions, around the first of January of 1998, in Sweden.

\begin{tabular}{lcc}
\hline Age group & Arriaga & Vaupel - Canudas \\
\hline $0-9$ & 0.013 & 0.013 \\
$10-19$ & -0.002 & -0.002 \\
$20-29$ & -0.001 & -0.001 \\
$30-39$ & 0.014 & 0.014 \\
$40-49$ & 0.020 & 0.020 \\
$50-59$ & 0.021 & 0.021 \\
$60-69$ & 0.045 & 0.045 \\
$70-79$ & 0.050 & 0.050 \\
$80-89$ & 0.025 & 0.025 \\
90 and above & 0.007 & 0.007 \\
\hline All ages & 0.191 & 0.191 \\
\hline
\end{tabular}

Source: Authors' calculations described in Note 1 and Note 2. Lifetable data are derived from the Human Mortality Database (2002). Lifetable values from the years 1995 and 2000 were used to obtain results for the first of January, 1998.

contribution to demographic research. ${ }^{3,4}$ 


\section{Notes}

1. If data are available for time $t$ and $t+h$, then we generally used the following approximations for the value at the mid-point $t+h / 2$. For the relative derivative of the function $v(a, t+h / 2)$, we used

$$
\dot{v}(a, t+h / 2) \approx \frac{\ln \left[\frac{v(a, t+h)}{v(a, t)}\right]}{h} .
$$

The value of the function at the mid-point $v(a, t+h / 2)$ was estimated by

$$
v(a, t+h / 2) \approx v(a, t) e^{(h / 2) \dot{v}(a, t+h / 2)} .
$$

Substituting the right-hand side of (48) for $v^{\prime}(a, t+h / 2)$ in (49) yields the equivalent approximation

$$
v(a, t+h / 2) \approx[v(a, t) v(a, t+h)]^{1 / 2} .
$$

This is a standard approximation in demography (Preston, Heuveline and Guillot, 2001). The derivative of the function $v(a, t+h / 2)$ was estimated by

$$
\dot{v}(a, t+h / 2)=\dot{v}(a, t+h / 2) v(a, t+h / 2) .
$$

We used (48)-(51) wherever we thought that the rate of change was more or less constant over the time interval. In some cases it seemed appropriate to assume that change in the interval was linear. This was the case when we estimated the change over time in the survivorship function $\ell(a, t)$ and life expectancy $e(a, t)$. Then we used

$$
v(a, t+h / 2) \approx \frac{v(a, t+h)+v(a, t)}{2}
$$

and

$$
\dot{v}(a, t+h / 2) \approx \frac{v(a, t+h)-v(a, t)}{h} .
$$

2. The mid-ages were calculated for the survivorship function $\ell(a, t)$ and the remaining life expectancy for each age group $e(a, t)$ following the formulas of 
Note 1. The period force of mortality in an interval, for all causes of death, was calculated using a formula similar to (48)

$$
\mu(a+h / 2, t) \approx \int_{a}^{a+h} \mu(x, t) d x=-\ln \left[\frac{\ell(a+h, t)}{\ell(a, t)}\right] .
$$

In Tables 2 and 3 the force of mortality for the $i$ th cause of death was estimated by multiplying the result of formula (54) by the proportion of deaths from cause $i, D_{i}(a+h / 2, t)$, in the total deaths of the age group, $D(a+h / 2, t)$,

$$
\mu_{i}(a+h / 2, t) \approx \mu(a+h / 2, t)\left[\frac{D_{i}(a+h / 2, t)}{D(a+h / 2, t)}\right] .
$$

The lifetable distribution of deaths from cause $i$ was calculated as

$$
f_{i}(a+h / 2, t) \approx \mu_{i}(a+h / 2, t) \ell(a+h / 2, t) .
$$

3. This article is in honor of Nathan Keyfitz's $90^{\text {th }}$ birthday. Nathan Keyfitz was born on June 29, 1913 in Montreal, Canada.

4. Let $\tilde{\rho}(t)$ denote the average value of the age-specific rate of progress in reducing death rates, $\rho(a, t)$, weighted by the the product of the remaining life expectancy $e^{o}(a, t)$ and the probability density function $f(a, t)$,

$$
\tilde{\rho}(t)=\frac{\int_{0}^{\omega} \rho(a, t) e^{o}(a, t) f(a, t) d a}{\int_{0}^{\omega} e^{o}(a, t) f(a, t) d a} .
$$

Then formula (15) implies that the change in life expectancy can be decomposed as:

$$
\dot{e}^{o}(0, t)=\tilde{\rho} e^{\dagger}
$$

We are in the process of applying (58) to analyze the dynamics of life expectancy. 


\section{References}

Andreev, E.M. 1982. "Method Komponent v Analize Prodoljitelnosty

Zjizni." [The Method of Components in the Analysis of Length of Life]. Vestnik Statistiki, 9: 42-47.

Andreev, Evgueni, Vladimir Shkolnikov and Alexander Z. Begun. 2002. "Algorithm for decomposition of differences between aggregate demographic measures and its application to life expectancies, Gini coefficients, health expectancies, parity-progression ratios and total fertility rates." Submitted to Demographic Research. Available at http://www.demogr.mpg.de/papers/working/wp-2002-035.pdf.

Arriaga, Eduardo E. 1984. "Measuring and Explaining the Change in Life Expectancies." Demography 21: 83-96.

Berkeley Mortality Database. University of California, Berkeley (USA). Available at www.demog.berkeley.edu/wilmoth/mortality/ (data downloaded on [18/Sep/2001])

Demetrius, Lloyd. 1979. "Relations Between Demographic Parameters." Demography 16: 329-338.

Goldman, Noreen and Graham Lord. 1986. "A New Look at Entropy and the Lifetable." Demography 23: 275-282.

Hill, Gerry. 1993. "The Entropy of the Survival Curve: An Alternative Measure." Canadian Studies in Population 20: 43-57.

Hokkert, Ralph. 1987. "Lifetable Transformations and Inequality Measures: Some Noteworthy Formal Relations." Demography 24: 615-622.

Human Mortality Database. University of California, Berkeley (USA), and Max Planck Institute for Demographic Research (Germany). Available at www.mortality.org or www.humanmortality.de (data downloaded on $[23 / 5 / 02])$.

Keyfitz, Nathan. 1977. "What Difference Does it Make if Cancer Were Eradicated? An Examination of the Taeuber Paradox." Demography 14: 411-418. 
—. 1985. Applied Mathematical Demography. 2nd ed. New York: Springer.

Mitra, S. 1978. "A Short Note on the Taeuber Paradox." Demography 15: 621-623.

Pollard, J. H. 1982. "The Expectation of Life and its Relationship to Mortality." Journal of the Institute of Actuaries 109: 225-240.

-. 1988. "On the Decomposition of Changes in Expectation of Life and Differentials in life Expectancy." Demography 25: 265-276.

Pressat, Roland. 1985. "Contribution des Écarts de Mortalité par Âge à la Différence des Vies Moyennes." Population 4-5: 765-770.

Preston, Samuel H., Patrick Heuveline and Michel Guillot. 2001.

Demography: Measuring and Modeling Population Processes. Oxford: Blackwell Publishers.

Vaupel, James W. 1986. "How Change in Age-Specific Mortality Affects Life Expectancy." Population Studies 40: 147-157.

—. 1992. "Analysis of Population Changes and Differences." Paper (107 pp.) presented at the PAA Annual Meeting held in Denver, Colorado, April 30 - May 21992.

Vaupel, James W. and Vladimir Canudas Romo. 2000. How Mortality Improvement Increases Population Growth. [in] Optimization, Dynamics and Economic Analysis: Essays in Honor of Gustav Feichtinger. Chapter Population Dynamics. Physica, Springer. p. 350-357. Available at http://www.demogr.mpg.de/Papers/Working/wp-1999-015.pdf.

—. 2002. "Decomposing Demographic Change into Direct vs. Compositional Components." Demographic Research 7: 1-14. Available at http://www.demographic-research.org/. 